\title{
Erratum: An Efficient Data Indexing Approach on Hadoop Using Java Persistence API
}

\author{
Yang Lai ${ }^{1,2}$ and Shi Zhong $\mathrm{Zhi}^{1}$ \\ ${ }^{1}$ The Key Laboratory of Intelligent Information Processing, Institute of Computing \\ Technology, Chinese Academy of Sciences, Beijing, 100190, China \\ ${ }^{2}$ Graduate University of Chinese Academy of Sciences, Beijing 100039, China \\ \{yanglai, shizz\}@ics.ict.ac.cn
}

Z. Shi et al. (Eds.): IIP 2010, IFIP AICT 340, pp. 213-224, 2010.

(C) IFIP International Federation for Information Processing 2010

\section{DOI 10.1007/978-3-642-16327-2_42}

In the original version, the first and last names of the authors are interchanged. They should read "Lai Yang, and ZhongZhi Shi". 